\title{
Blockade of sonic hedgehog signaling decreases viability and induces apoptosis in retinoblastoma cells: The key role of the PI3K/Akt pathway
}

\author{
ZHIDU SONG ${ }^{1}$, YUANYUAN DU ${ }^{1}$ and YING TAO ${ }^{2}$ \\ ${ }^{1}$ Department of Ophthalmology, The Second Hospital of Jilin University, Changchun, Jilin 130041; ${ }^{2}$ Department \\ of Anesthesiology, The Third Hospital of Jilin University, Changchun, Jilin 130033, P.R. China
}

Received June 2, 2016; Accepted March 23, 2017

DOI: $10.3892 / \mathrm{ol} .2017 .6701$

\begin{abstract}
Retinoblastoma (RB) is the most common type of malignant intraocular cancer in teenagers. One of the proteins abnormally expressed during oncogenesis of $\mathrm{RB}$ is sonic hedgehog ( $\mathrm{SHH})$, which possesses the capability to selectively activate transcription factors of different genes. However, the detailed function of $\mathrm{SHH}$ in $\mathrm{RB}$ remains unknown. Thus, the present study sought to investigate the role of $\mathrm{SHH}$ in the development of RB. The human RB WERI-Rb-1 cell line was used as an in vitro model for the knockdown of $\mathrm{SHH}$ by a specific short hairpin RNA (shRNA). To assess the effect of SHH inhibition on cell growth and apoptosis, cell viability, colony formation and flow cytometry assays were conducted. WERI-Rb-1 cells transfected with an shRNA targeting $\mathrm{SHH}$ were treated with the phosphoinositide-3 kinase (PI3K)/Akt agonist insulin-like growth factor 1 (IGF-1) to investigate the possible mechanism by which $\mathrm{SHH}$ promotes RB. The present results revealed that the silencing of $\mathrm{SHH}$ induced G1 cell-cycle arrest and apoptosis in WERI-Rb-1 cells and led to a decrease in cell viability, indicating that $\mathrm{SHH}$ has a critical role in the determination of RB cell survival. Moreover, according to the results of the IGF-1 assays, suppression of PI3K/Akt was a prerequisite for $\mathrm{SHH}$ inhibition, illuminating its potential role in the treatment of RB. The findings outlined in the present study elucidate a clear link between SHH and the PI3K/Akt pathway in RB cell survival, which could provide valuable inspiration for the advancement of therapies against RB.
\end{abstract}

\section{Introduction}

Retinoblastoma (RB) is the most common type of malignant intraocular cancer in children $<5$ years old (1). In the majority of

Correspondence to: Dr Ying Tao, Department of Anesthesiology, The Third Hospital of Jilin University, 829 Xinmin Avenue, Changchun, Jilin 130033, P.R. China

E-mail: taoying_12312@sina.com

Key words: apoptosis, Akt, phosphoinositde-3 kinase, retinoblastoma, sonic hedgehog cases, particularly in developing countries, RB is only detected at the late stages of the cancer, which severely threatens the lives of the patients (2). Prior to the 1990s, surgical removal of the afflicted eyes, followed by external-beam radiotherapy, was the major treatment method for RB; however, the approach was associated with high morbidity and craniofacial deformity (3). At present, the most effective therapy against $\mathrm{RB}$ is chemotherapy combined with immunotherapy, which is cytotoxic to RB cells, increasing cell apoptosis (2). Despite its high efficacy, the current therapy remains somewhat unsatisfactory, owing to the adverse effects of chemotherapy. Thus, the development of mild therapeutic modalities to improve the outcome and survival of RB is required.

Numerous signaling molecules are relevant to the oncogenesis and development of cancer. One of these signaling transduction pathways is the hedgehog $(\mathrm{HH})$ pathway, which governs processes such as embryonic development, cell differentiation, cell proliferation and tissue patterning (4). The most studied member of the vertebrate $\mathrm{HH}$ family is sonic hedgehog ( $\mathrm{SHH})$. SHH exerts its function by selectively activating the transcription factors of different genes. Altered SHH pathway activation has been observed in different types of solid and non-solid cancer (5). The selective regulation of SHH production can influence cell apoptosis and the metastasis of pancreatic and ovarian cancer cells through multiple mechanisms (6-8). These findings reveal the close connection between the SHH pathway and other critical pathways, which include phosphoinositide-3 kinase (PI3K)/Akt and nuclear factor $\kappa$-light-chain-enhancer of activated $B$ cells pathways. Considering that tumorigenesis initiates aberrant apoptotic processes, the aforementioned data suggest that the SHH pathway may exert control over apoptosis in cancer cells. Moreover, a recent study regarding the $\mathrm{SHH}$ pathway has validated the critical role of the $\mathrm{SHH}$ pathway in the progression of RB (9). From a therapeutic point of view, it is therefore desirable to further evaluate how regulation of the SHH pathway affects RB cell survival.

In the current study, the expression of $\mathrm{SHH}$ in the human RB WERI-Rb-1 cell line was regulated using specific short hairpin RNAs (shRNAs). Cell viability and apoptosis were measured to investigate the function of SHH in the survival of RB cells. As PI3K/Akt serves such a central role in cell 
apoptosis, further experiments subjecting WERI-Rb-1 cells to insulin-like growth factor 1 (IGF-1) were conducted to assess the possible mechanism by which $\mathrm{SHH}$ drives the promotion of RB. IGF-1 is a pleiotropic polypeptide with a wide range of actions in the central and peripheral nervous systems, which protects neurons against cell death induced by amyloidogenic derivatives, glucose or serum deprivation via the activation of intracellular pathways implicating PI3K/Akt (10). Inhibition of SHH significantly increased the apoptotic rate in the WERI-Rb-1 cells and resulted in a decrease in cell viability. Downregulation of PI3K/Akt is therefore required for $\mathrm{SHH}$ inhibition-based anti-RB therapies. The findings in the present study demonstrate a clear interaction between $\mathrm{SHH}$ and the PI3K/Akt pathway in the survival of RB cells and could aid the development of therapies for the prevention of this malignancy.

\section{Materials and methods}

Chemicals and cell culture. Antibodies against SHH and phosphorylated (p)-PI3K (cat. nos. bs-1544R and bs-5538R, respectively) were purchased from Beijing Biosynthesis Biotechnology Co., Ltd. (Beijing, China). Antibodies against cleaved caspase-3 and cleaved poly (ADP-ribose) polymerase (PARP) (cat. nos. ab2302 and ab32561, respectively) were purchased from Abcam (Cambridge, MA, USA). Antibodies against B-cell CLL/lymphoma 2 (Bcl-2), Bcl-2 associated X (Bax) and PI3K (cat. nos. BA0412, BA0315 and BA1352, respectively) were purchased from Boster Systems, Inc. (Pleasanton, CA, USA). Antibodies against p-Akt (Ser 473), Akt and $\beta$-actin (cat. nos. sc-8312, sc-135651 and sc-47778, respectively) were purchased from Santa Cruz Biotechnology, Inc. (Dallas, TX, USA). The secondary IgG antibodies conjugated to horseradish peroxidase (cat. nos. WLA023 and WLA024) were purchased from Wanleibio Co., Ltd. (Shanghai, China). The PI3K/Akt agonist IGF-1 (cat. no. 10598-R023) was obtained from Sino Biological Inc. (Beijing, China). Transfection agents (cat. no. c1507) were purchased from Applygen Technologies Inc. (Beijing, China). A specific $S H H$-targeted shRNA (5'-CCCGACATCATATTTAAGGAT-3') and control shRNA (5'-GCTGTTGGACAGCGAGACCAT-3') were constructed by Wanlei Life Sciences (Shenyang, China) in accordance with a previous study (11). The human RB WERI-Rb-1 cell line was obtained from Wanlei Life Sciences and cultured in RPMI-1640 medium (cat. no. 31800-014; Gibco; Thermo Fisher Scientific, Inc., Waltham, MA, USA) supplemented with $20 \%$ fetal bovine serum (FBS; cat. no. SH30084.03; HyClone; GE Healthcare Life Sciences, Logan, UT, USA) and a $1 \%(\mathrm{v} / \mathrm{v})$ antibiotics mixture (penicillin/streptomycin; cat. no. E485-20ML; Amresco, LLC, Solon, OH, USA) in an atmosphere of $95 \%$ air and $5 \% \mathrm{CO}_{2}$ at $37^{\circ} \mathrm{C}$.

Knockdown of SHH gene in WERI-Rb-1 cells. Sequences of different shRNAs $(1.2 \mu \mathrm{g} / 100 \mu \mathrm{l})$ were ligated into pRNAH1.1 plasmid (Sangon Biotech, Co., Ltd., Shanghai, China) to form transfection vectors, and transfection was conducted using HiGene transfection reagent (cat. no. C1507) from Applygen Technologies Inc., according to the manufacturer's protocol. Cells were divided into three groups: A control group of untransfected WERI-Rb-1 cells; an shNC group of WERI-Rb-1 cells transfected with a pRNAH1.1-NC (non-targeted shRNA) plasmid; and an shSHH group of WERI-Rb-1 cells transfected with a pRNAH1.1-SHH (SHH-targeted shRNA) plasmid. Each treatment was performed in triplicate. Stable transfected cells were screened in RPMI-1640 medium in the presence of $\mathrm{G} 418(0.5 \mu \mathrm{g} / \mu \mathrm{l})$ for subsequent experiments. The knockdown efficiency of $\mathrm{SHH}$ by shRNA was determined using reverse transcription-quantitative PCR (RT-qPCR) and western blot analysis.

$R T-q P C R$. For the detection of mRNA, whole RNAs in cells under different conditions were extracted using an RNA extraction kit according to the manufacturer's instructions (cat. no. RP1201; BioTeke Corporation, Beijing, China). $\beta$-actin was selected as the internal reference gene. cDNA templates of $\mathrm{SHH}$ were attained by reverse transcribing the RNA using an RT-PCR kit (cat. no. PR6502; BioTeke Corporation), and the final RT-qPCR reaction mixture of volume $20 \mu \mathrm{l}$ contained $10 \mu \mathrm{l}$ SYBR Green master mix, $0.5 \mu \mathrm{l}$ of each primer $(10 \mu \mathrm{M})$ (SHH, forward, 5'-AGCAACAGCAACGCAAAG-3' and reverse, 5'-AATAGCCAGGAGAGGAGGA-3'; and $\beta$-actin forward, 5'-TTAGTTGCGTTACACCCTTTCTTG-3' and reverse, 5'-CTGTCACCTTCACCGTTCCAGTTT-3'), $1 \mu \mathrm{l}$ of the cDNA template and $8 \mu \mathrm{l}$ RNase-free $\mathrm{H}_{2} \mathrm{O}$. Thermal cycling parameters for the amplification were as follows: A denaturation step at $95^{\circ} \mathrm{C}$ for $10 \mathrm{~min}$, followed by 40 cycles of $95^{\circ} \mathrm{C}$ for $10 \mathrm{sec}, 60^{\circ} \mathrm{C}$ for $20 \mathrm{sec}$ and $72^{\circ} \mathrm{C}$ for $30 \mathrm{sec}$. The relative expression level of the targeted gene was calculated with Data Assist Software version 3.0 (Applied Biosystems; Thermo Fisher Scientific, Inc.) according to the $2^{-\Delta \Delta \mathrm{Cq}}$ method (12). Each assay was repeated $\geq 3$ times.

Western blotting assay. The whole protein product in each group was extracted using Whole Protein Extraction kit according to the manufacturer's instructions (Wanleibio Co., Ltd.). $\beta$-actin was used as an internal reference protein. Protein concentration was determined using a bicinchoninic acid assay. Next, $40 \mu \mathrm{g}$ protein was subject to SDS-PAGE with a $10 \%$ gel. Following the transfer of targeted proteins onto polyvinylidene difluoride sheets, the membranes were washed with Tris-buffered saline plus Tween 20 (TTBS; cat. no. WLA025; Wanleibio Co., Ltd.) for 5 min and then blocked with skimmed milk powder solution for $1 \mathrm{~h}$. Primary antibodies against $\mathrm{SHH}$ (1:500), cleaved caspase-3 (1:1,000), cleaved-PARP $(1: 1,000)$, Bcl-2 (1:400), Bax (1:400), p-PI3K (1:500), PI3K (1:400), p-Akt $(1: 200)$, Akt $(1: 200)$ or $\beta$-actin $(1: 1,000)$ were added and the membranes were incubated at $4{ }^{\circ} \mathrm{C}$ overnight. After an additional four washes with TTBS, secondary IgG antibodies conjugated to horseradish peroxidase $(1: 5,000)$ were incubated with the membranes for $45 \mathrm{~min}$ at $37^{\circ} \mathrm{C}$. Following a further six washes with TTBS, the blots were developed using Beyo ECL Plus reagent (Beyotime Institute of Biotechnology, Haimen, China) and the results were recorded in the Gel Imaging System (cat. no. WD-9413B; Beijing Liuyi Instrument Factory, Beijing, China). The relative expression levels of $\mathrm{SHH}$ in different groups were calculated with the Gel-Pro-Analyzer (Media Cybernetics, Inc., Rockville, MD, USA).

3-(4,5-dimethylthiazol-2-yl)-2,5-diphenyltetrazolium bromide (MTT) test. The viability of different groups of WERI-Rb-1 
cells was measured using an MTT assay. A total of $50 \mu 1$ exponentially growing cells $\left(4 \times 10^{3}\right.$ cells $\left./ \mathrm{ml}\right)$ were seeded into a 96 -well plate and cultured at $37^{\circ} \mathrm{C}$ for $120 \mathrm{~h}$. For each group, the cell viabilities at 24, 48, 72, 96 and $120 \mathrm{~h}$ were all determined and each time point was represented by five replicates. In brief, $5 \mathrm{mg} / \mathrm{ml}$ MTT was added to each well. After incubating for an additional $4 \mathrm{~h}$ at $37^{\circ} \mathrm{C}, 200 \mu \mathrm{l}$ dimethyl sulfoxide was added and the cell viabilities of different groups were measured with a microplate reader at $490 \mathrm{~nm}$.

Flow cytometry. WERI-Rb-1 cells in different groups were cultured in RPMI-1640 medium supplemented with 10\% FBS at $37^{\circ} \mathrm{C}$ and collected by centrifugation at $626 \mathrm{x}$ for $5 \mathrm{~min}$ at $37^{\circ} \mathrm{C}$. A flow cytometry assay was conducted to determine the effect of $\mathrm{SHH}$-knockdown on the cell-cycle distribution of WERI-Rb-1 cells. In brief, cells were fixed with $70 \%$ ethanol at $4^{\circ} \mathrm{C}$ for $2 \mathrm{~h}$. A total of $500 \mu \mathrm{l}$ propidium iodide (PI)-fluorescein isothiocyanate (FITC) $(2 \mu \mathrm{g} / \mathrm{ml})$ was then added to different samples to stain DNA in the dark at $4^{\circ} \mathrm{C}$ for $30 \mathrm{~min}$. After a 20-min incubation at room temperature, the DNA contents of the cells were analyzed using a flow cytometer (Accuri C6; BD Biosciences, Franklin Lakes, NJ, USA).

Colony formation in soft agar. The capability of cancer cells for anchorage-independent growth was measured by colony formation assay. Cells that had been transfected with different plasmids were seeded into RPMI-1640 medium containing 3\% methylcellulose at a concentration of 500 cells per plate. After 3 weeks in culture, the numbers of colonies were counted using an inverted phase contrast microscope (cat. no. AE31; Motic Incorporation, Ltd., Causeway Bay, Hong Kong). Colony formation rate $=$ colony number/inoculation cell number $\mathrm{x} 100$.

Determination of the effect of SHH-knockdown on the apoptotic process in WERI-Rb-1 cells. To determine the effect of $S H H$-knockdown on the apoptotic process of WERI-Rb-1 cells, cells were first probed with an Annexin V-FITC Apoptosis Detection kit (Wanleibio Co., Ltd.) to determine the apoptotic rates according to the manufacturer's protocol. In brief, $5 \mu \mathrm{l}$ annexin $\mathrm{V}$ was added to different wells. Following incubation with annexin $\mathrm{V}$ for $10 \mathrm{~min}$ at room temperature, the cells were resuspended with $1 \mathrm{X}$ binding buffer (Annexin V-FITC Apoptosis Detection kit) and $5 \mu \mathrm{l}$ PI was added. Next, the apoptotic rates were analyzed using FACScan flow cytometry (Accuri C6; BD Biosciences, Franklin Lakes, NJ, USA). The apoptotic cell rate [upper right quadrant advanced-stage apoptosis cell percentage (UR) + lower right quadrant prophase apoptosis cell percentage (LR)-whole apoptosis cell percentage] was equal to the sum of the late apoptotic rate (UR) and the early apoptotic rate (LR). Additionally, the morphological changes of the nuclei in different groups were detected using a Hoechst staining kit, according to the manufacturer's instructions (Beyotime Institute of Biotechnology) and the results were recorded using a fluorescence microscope (IX53; Olympus Corporation, Tokyo, Japan) under x400 magnification. Cells with nuclei stained bright blue were judged to be Hoechst-positive and undergoing apoptosis. The expression of apoptosis-associated molecules, including cleaved caspase-3, cleaved PARP, Bcl-2, Bax, p-PI3K, PI3K, p-Akt and Akt, was determined by western blot analysis, as aforementioned.
Determination of effect of SHH-knockdown on the PI3K/Akt pathway in WERI-Rb-1 cells. To explain the mechanism driving the function of $S H H$ in RB, WERI-Rb-1 cells in shNC and $\mathrm{shSHH}$ groups were administered with $1 \mu \mathrm{g} / 10 \mu \mathrm{l}$ of the PI3K/Akt agonist IGF-1 for $48 \mathrm{~h}$. In total, four groups were set up: The NC group, with shNC-transfected WERI-Rb-1 cells; the shSHH group, with shSHH-transfected WERI-Rb-1 cells; the NC+IGF-1 group, with shNC-transfected cells treated with IGF-1; and the shSHH+IGF-1 group, with shSHH-transfected cells treated with IGF-1. Following incubation, the cell viability, apoptotic rates and expression of $\mathrm{p}-\mathrm{PI} 3 \mathrm{~K}, \mathrm{PI} 3 \mathrm{~K}$, p-Akt, Akt, cleaved caspase-3, cleaved PARP, Bcl-2 and Bax were determined as aforementioned.

Statistical analysis. All statistical analysis and graph manipulation was conducted using R version 3.2.1 (https://cran.r-project .org/doc/FAQ/R-FAQ.html\#Citing-R). Data are expressed as the mean \pm standard deviation. Differences between multiple groups were determined based on the least-significant difference method, with $\mathrm{P}<0.05$ considered to indicate a significant difference.

\section{Results}

Transfection of specific shRNA reduces the production of $\mathrm{SHH}$ at $m R N A$ and protein levels. Transfection of WERI-Rb-1 cells with $\mathrm{SH} H$-specific shRNA markedly attenuated the expression of SHH at the mRNA and protein levels, and the difference between the shSHH group and the control or shNC groups was statistically different (Fig. 1; both $\mathrm{P}<0.05$ ).

Knockdown of SHH gene decreases cell viability and anchorage independent growth ability of WERI-Rb-1 cells. The cell viability of the different groups were measured using an MTT assay. The transfection of $S H H$-specific shRNA reduced the viability of the RB cells (Fig. 2A). The negative effect of shRNA transfection on cell viability could be observed from $48 \mathrm{~h}$ after incubation, and the difference between the shSHH group and the control or shNC groups was statistically different at the last four sampling time points (all $\mathrm{P}<0.05$ ). Additionally, the anchorage-independent growth ability of WERI-Rb-1 cells was markedly decreased by knockdown of $\mathrm{SHH}$ (Fig. 2B). The colony formation rate of cells in the shSHH group $(20.7 \pm 2.2 \%)$ was almost half of those in the control $(44.2 \pm 4.7 \%)$ and $\operatorname{shNC}(41.1 \pm 4.4 \%)$ groups, and the difference was statistically significant (both $\mathrm{P}<0.05$ ).

Knockdown of SHH gene induces G1 cell cycle arrest and apoptosis in WERI-Rb-1 cells. The cell-cycle distribution and apoptosis of cells under different conditions were analyzed with flow cytometry. The cell-cycle distribution analysis demonstrated a G1 cell-cycle arrest in WERI-Rb-1 cells administered with $\mathrm{SHH}$-specific shRNA (Fig. 3A). The percentage of cells arrested in the G1 phase in the shSHH group $(75.4 \pm 5.0 \%)$ was markedly higher than those in control $(58.9 \pm 5.8 \%)$ and shNC $(58.3 \pm 5.6 \%)$ groups. The downregulation of $\mathrm{SHH}$ also concomitantly decreased the fraction of cells in the $\mathrm{S}$ phase, representing a halt in cell proliferation through G1-phase cell-cycle arrest. Consistent with the results of cell-cycle distribution, the cell apoptotic rate in the shSHH 

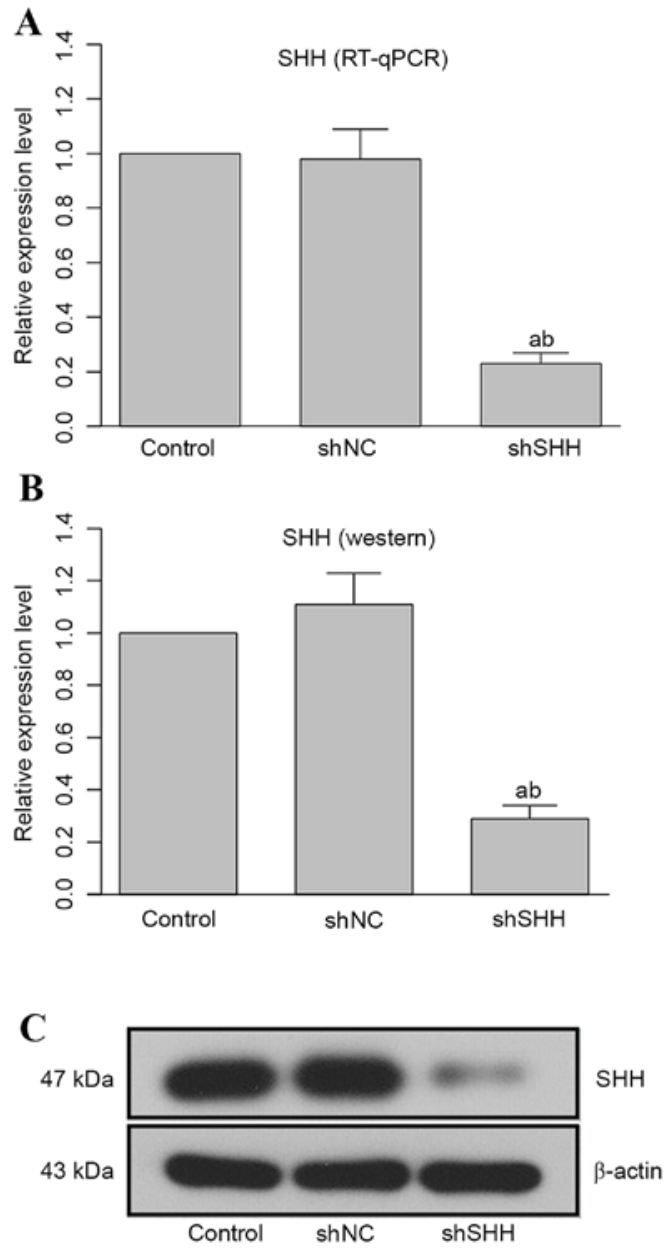

Figure 1. Validation of $S H H$-knockdown efficiency by RT-qPCR and western blotting assay. (A) Quantitative analysis result of RT-qPCR validation of $S H H$-knockdown efficiency. (B) Quantitative analysis result showing $S H H$-knockdown efficiency. (C) Representative image of western blot analysis, confirming the knockdown of $S H H ; \beta$-actin was used as a control. ${ }^{a} \mathrm{P}<0.05$ vs. control group; ${ }^{b} \mathrm{P}<0.05$ vs. shNC group. $\mathrm{SHH}$, sonic hedgehog; RT-qPCR, reverse transcription-quantitative polymerase chain reaction; shNC, non-targeting short hairpin RNA.

group was also significantly higher than those in the other two groups $(\mathrm{P}<0.05$; Fig. 3B). The morphological alteration of cell nuclei in different groups was observed with Hoechst staining. More Hoechst-positive nuclei were recorded in the shSHH group than in the control or shNC groups (Fig. 3C). To further reveal the function of SHH in the induction of apoptosis in WERI-Rb-1 cells, the expression of indicators associated with cell apoptosis was analyzed: It was found that the expression of pro-apoptotic molecules (cleaved caspase-3, cleaved PARP and Bax) was upregulated, whereas the expression of anti-apoptotic molecules (Bcl-2, p-PI3K and p-Akt) was downregulated (Fig. 3D). On the basis of quantitative analyses, the difference in relative expression level between the shSHH group and the control or shNC groups was statistically different for these 6 groups (Fig. 3D; $\mathrm{P}<0.05$ ).

Knockdown of SHH gene inhibits the activation of the PI3K/Akt pathway. The effect of $S H H$-knockdown on the PI3K/Akt pathway was further analyzed by administering cells in the shNC and shSHH groups with IGF-1. IGF-1 treatment
A

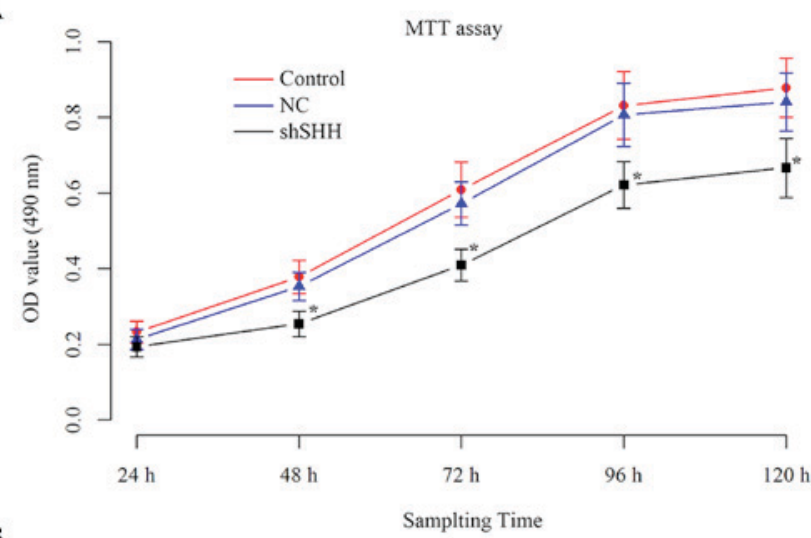

B
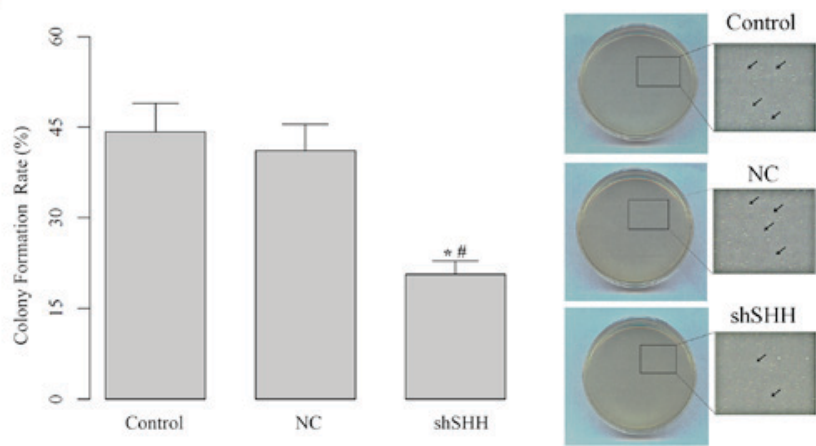

Figure 2. Knockdown of $S H H$ inhibits the viability and anchorage-dependent growth ability of WERI-Rb-1 cells. (A) Quantitative analysis results of MTT assay. (B) Quantitative analysis result and representative images of colony formation assay. Arrows indicate colonies. ${ }^{a} \mathrm{P}<0.05$ vs. control group; ${ }^{b} \mathrm{P}<0.05$ vs. shNC group. SHH, sonic hedgehog; MTT, 3-(4,5-dimethylthiazol-2-yl)-2,5-diphenyltetrazolium bromide; shNC, non-targeting short hairpin RNA; OD, optical density.

increased cell viability, which was attenuated by transfection with shSHH (Fig. 4A). However, the difference between the untreated shSHH and shSHH+IGF-1 groups was not statistically significant $(\mathrm{P}>0.05)$. Contrary to the results of the MTT assay, increased apoptosis induced by knockdown of $\mathrm{SHH}$ was markedly inhibited by IGF-1; the difference between the untreated shSHH and shSHH+IGF-1 groups was statistically significant $(\mathrm{P}<0.05$; Fig. 4B). Moreover, following administration of IGF-1, the expression patterns of apoptosis-related molecules in the shSHH+IGF-1 group were reversed with regards to the control when compared with the untreated shSHH group (Fig. 4C).

\section{Discussion}

Recent studies have demonstrated that levels of SHH are elevated in multiple types of human cancer (13-17), which subsequently results in the aberrant activation of the $\mathrm{HH}$ signaling cascade. Thus, the SHH-dependent initiation of the $\mathrm{HH}$ pathway has been hypothesized to be a critical mechanism for the promotion of tumor cell viability. Emerging research based on different types of cancer has validated this hypothesis by revealing multiple important pro-cancer pathways associated with the dysregulated expression of $\operatorname{SHH}(7,8,18)$. Consequently, the role of SHH in RB was investigated in the present study, the major finding of which was that knockdown of $\mathrm{SHH}$ decreased the proliferation of RB cells and induced 
A
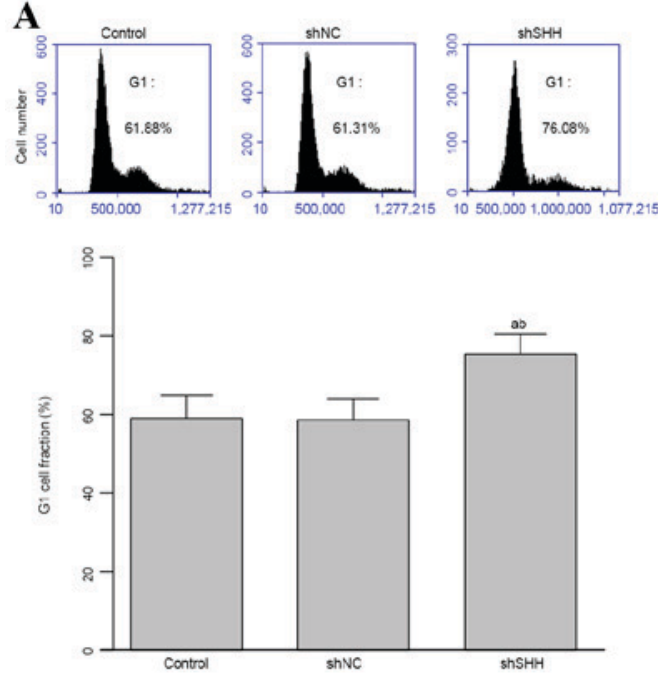

B
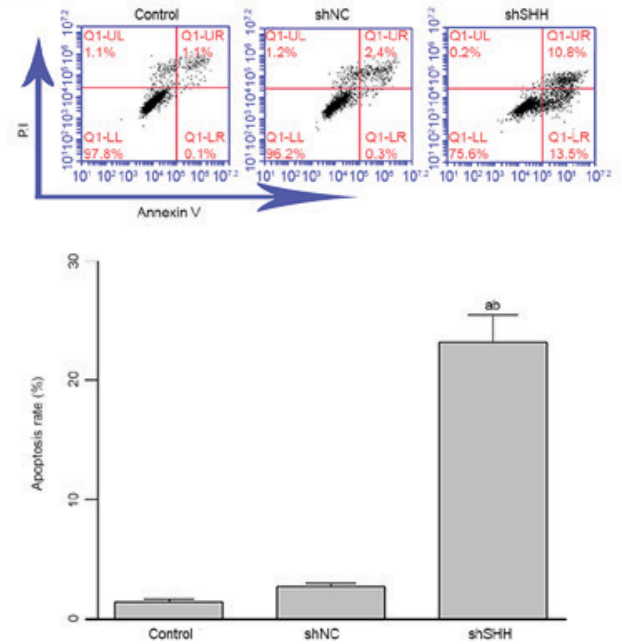

C
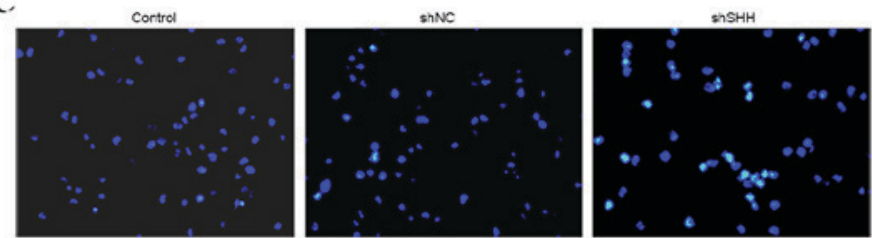

D
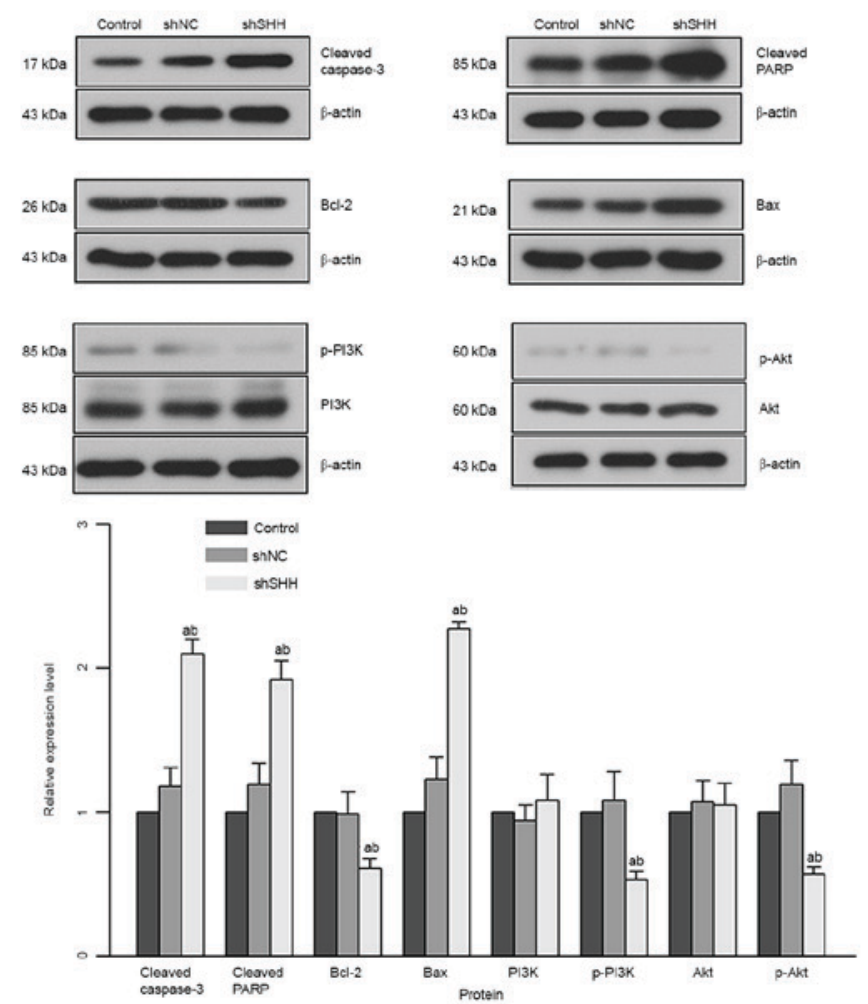

Figure 3. Knockdown of $\mathrm{SHH}$ induces cell-cycle arrest and apoptosis in WERI-Rb-1 cells. (A) Representative image and quantitative analysis of cell-cycle distribution. (B) Representative image and quantitative analysis result of apoptosis. (C) Representative images of Hoechst staining; nuclei of cells in shSHH group were stained bright blue and abnormally shaped (x400 magnification). (D) Representative image and quantitative analysis result of western blotting assay. ${ }^{a} \mathrm{P}<0.05$ vs. control group; ${ }^{\text {P }}<0.05$ vs. shNC group. SHH, sonic hedgehog; shSHH, short hairpin RNA targeting SHH; shNC, non-targeting short hairpin RNA; Bcl-2, B-cell chronic lymphocytic lymphoma 2; PARP, poly (ADP-ribose) polymerase; Bax, Bcl-2-associated X; p-PI3K, phosphorylated phosphoinositide-3 kinase; PI, phosphoinositide.

apoptosis. Moreover, the effect of SHH in RB was mediated through a PI3K/Akt-dependent manner.
Choe et al (9) verified the aberrant expression of SHH in patients with RB and identified SHH as potential therapeutic 
$\mathbf{A}$

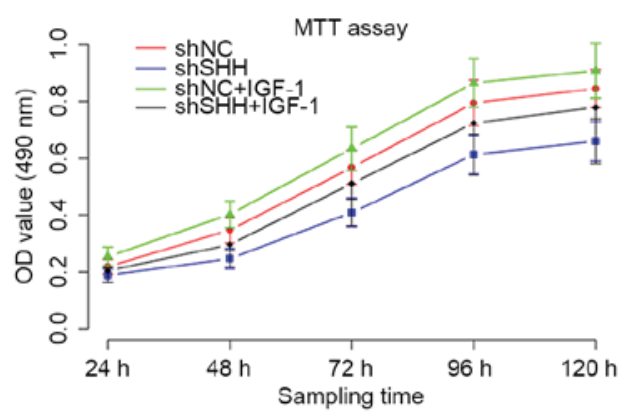

C

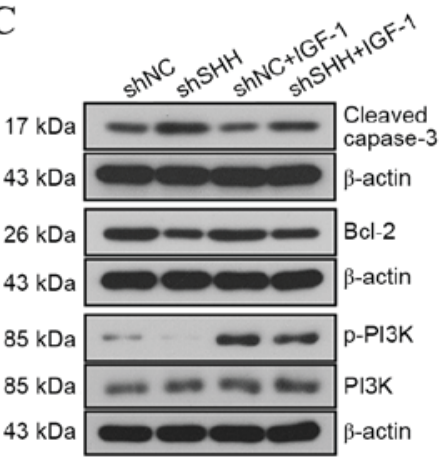

B

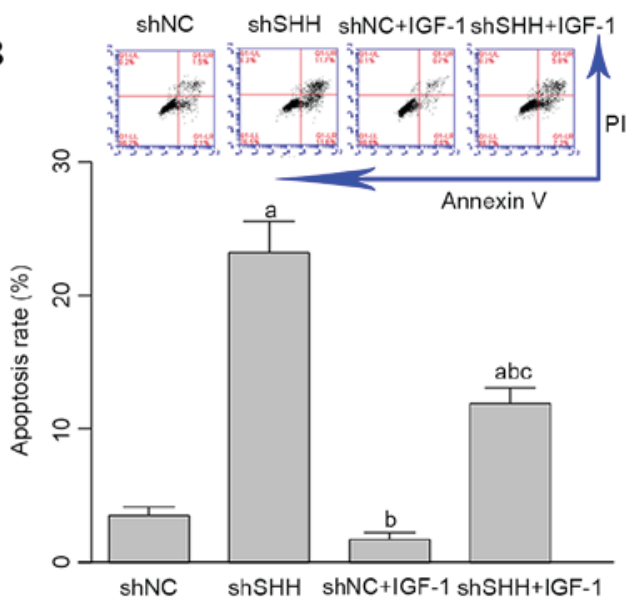

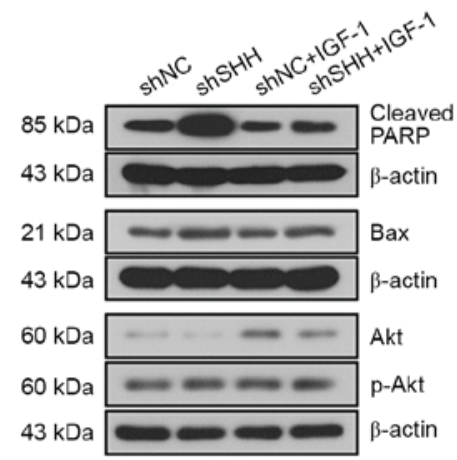

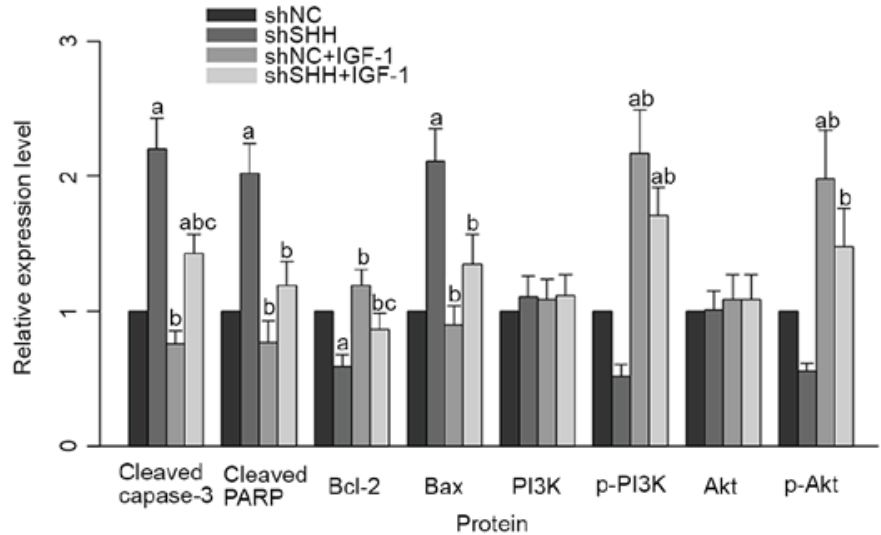

Figure 4. Administration of PI3K/Akt agonist IGF-1 increases cell viability and inhibits apoptosis in WERI-Rb-1 cells. (A) Quantitative analyses results of MTT assay. (B) Representative image and quantitative analysis result of western blotting assay; the expression of anti-apoptosis molecules (Bcl-2, p-PI3K and p-Akt) was upregulated, while the expression of pro-apoptosis molecules (cleaved caspase 3, cleaved PARP and Bax) was inhibited. (C) Representative image and quantitative analysis result of apoptosis. ${ }^{a} \mathrm{P}<0.05$ vs. shNC group; ${ }^{b} \mathrm{P}<0.05$ vs. shSHH group; ${ }^{\mathrm{c}} \mathrm{P}<0.05$ vs. shNC+IGF- 1 group. $\mathrm{p}$-PI3K, phosphorylated phosphoinositide-3 kinase; IGF-1, insulin-like growth factor 1; MTT, 3-(4,5-dimethylthiazol-2-yl)-2,5-diphenyltetrazolium bromide; Bcl-2, B-cell chronic lymphocytic lymphoma 2; PARP, poly (ADP-ribose) polymerase; Bax, Bcl-2-associated X; shSHH, short hairpin RNA targeting SHH; shNC, non-targeting short hairpin RNA; OD, optical density.

target for RB treatment. However, the study failed to provide a comprehensive mechanism to underpin these findings. Indicating that $\mathrm{SHH}$ is involved in oncogenesis and the development of RB, the expression of $S H H$ was downregulated by a $S H H$-specific shRNA in the human RB WERI-Rb-1 cell line in the current study. Following transfection, the cell viability and anchorage-independent growth ability were markedly decreased. Indeed, the anchorage-independent growth ability was representative of the oncogenicity of cells. Thus, it can be inferred that the knockdown of $\mathrm{SHH}$ evidently reduces the ability of RB to transform into a malignancy. SHH serves a central role in embryonic development and controls key genes that modulate cell proliferation (19) directly and indirectly $(4,20)$. During carcinogenesis, SHH exerts its function in a similar pattern to normal development and organ growth, but this aberrant signaling activity promotes the recapitulation of the development ability in cancer cells.

In addition, in the present study, expression of $\mathrm{SHH}$ not only promoted the growth of RB cells, but also inhibited apoptosis in RB cells. As illustrated by flow cytometry, 
knockdown of SHH induced G1 cell-cycle arrest and apoptosis in the WERI-Rb-1 cell line. At the protein level, the expression of certain apoptosis-related indicators was also detected. Pro-apoptotic factors, including cleaved caspase-3, cleaved PARP and Bax, were all upregulated following $\mathrm{SHH}$ shRNA transfection. By contrast, the activity of anti-apoptotic factors, including Bcl-2, PI3K and Akt, were inhibited when SHH expression was knocked down. As aforementioned, SHH-related pathways do not always proceed directly, but rather induce a complex network of cascades that cross-talk with other pathways to successfully exert a role in different biological processes $(21,22)$. The PI3K/Akt signal transduction pathway is closely associated with SHH. Previous studies have shown that SHH can promote the epithelial-mesenchymal transition (EMT) in ovarian cancer, and promote metastasis and lymphangiogenesis in gastric cancer in a PI3K/Akt dependent manner $(8,18)$. Riobó et al (23) suggested that PI3K/Akt has a synergistic function in the SHH signaling in embryonic development and cancer. To investigate further the interaction between PI3K/Akt and SHH in RB, WERI-Rb-1 cells that had been transfected with $S H H$-specific shRNA were treated with IGF-1 in the present study. This treatment significantly increased the expression of anti-apoptotic molecules, while decreasing the apoptotic rate of $\mathrm{SHH}$-knockdown RB cells. These results clearly demonstrated that the activation of the PI3K/Akt pathway blocked the antitumor effect of $S H H$-knockdown. Prior investigation suggests that it is possible that the effect of $\mathrm{PI} 3 \mathrm{~K} / \mathrm{Akt}$ pathway on SHH signaling is modulated through the enhancement of Gli-dependent transcriptional activity (18), which requires further study. However, the role of PI3K/Akt in regulating the SHH pathway varies between cell types, as $\mathrm{PI} 3 \mathrm{~K} / \mathrm{Akt}$ is critical for SHH-mediated EMT and metastasis in multiple cancer types (18). Therefore, the suppression of the PI3K/Akt pathway is key for the development of SHH-based anti-RB therapies. It is thus recommended that treatment modalities targeting SHH signaling should take into account the influence on the PI3K/Akt pathway.

In conclusion, the results of the present study suggest that SHH signaling plays an important role in the development of $\mathrm{RB}$, and that knockdown of $S H H$ could decrease cell growth and induce apoptosis in RB cells. At the molecular level, the function of SHH was found to closely associate with the activation of the PI3K/Akt pathway. However, the present study did not detect a change in $S H H$ level following administration of IGF-1 and could not provide a detailed explanation for the response of SHH expression to the regulation of the PI3K/Akt pathway. Thus, further studies should be conducted to reveal further the SHH-associated signaling transduction during RB.

\section{References}

1. Devesa SS: The incidence of retinoblastoma. Am J Ophthalmol 80: 263-265, 1975.

2. Liu Q, Wang Y, Wang H, Liu Y, Liu T and Kunda PE: Tandem therapy for retinoblastoma: Immunotherapy and chemotherapy enhance cytotoxicity on retinoblastoma by increasing apoptosis J Cancer Res Clin 139: 1357-1372, 2013.

3. Broaddus E, Topham A and Singh AD: Survival with retinoblastoma in the USA: 1975-2004. Brit J Ophthalmol 93: 24-27, 2009.
4. Milla LA, González-Ramírez CN and Palma V: Sonic Hedgehog in cancer stem cells: A novel link with autophagy. Biol Res 45: 223-230, 2012.

5. Katoh Y and Katoh M: Hedgehog target genes: Mechanisms of carcinogenesis induced by aberrant hedgehog signaling activation. Curr Mol Med 9: 873-886, 2009.

6. Feldmann G, Dhara S, Fendrich V, Bedja D, Beaty R, Mullendore M, Karikari C, Alvarez H, Iacobuzio-Donahue C, Jimeno A, et al: Blockade of hedgehog signaling inhibits pancreatic cancer invasion and metastases: A new paradigm for combination therapy in solid cancers. Cancer Res 67: 2187-2196, 2007.

7. Kasperczyk H, Baumann B, Debatin KM and Fulda S: Characterization of sonic hedgehog as a novel NF-kappaB target gene that promotes NF-kappaB-mediated apoptosis resistance and tumor growth in vivo. FASEB J 23: 21-33, 2009.

8. Ke Z, Caiping S, Qing Z and Xiaojing W: Sonic hedgehog-Gli1 signals promote epithelial-mesenchymal transition in ovarian cancer by mediating PI3K/AKT pathway. Med Oncol 32: 368, 2015.

9. Choe JY, Yun JY, Jeon YK, Kim SH, Choung HK, Oh S, Park M and Kim JE: Sonic hedgehog signalling proteins are frequently expressed in retinoblastoma and are associated with aggressive clinicopathological features. J Clin Pathol 68: 6-11, 2015.

10. Zheng WH, Kar S, Doré S and Quirion R: Insulin-like growth factor-1 (IGF-1): A neuroprotective trophic factor acting via the Akt kinase pathway. J Neural Transm Suppl: 261-272, 2000.

11. Hung HC, Hsiao YH and Gean PW: Sonic hedgehog signaling regulates amygdalar neurogenesis and extinction of fear memory. Eur Neuropsychopharmacol 25: 1723-1732, 2015.

12. Livak KJ and Schmittgen TD: Analysis of relative gene expression data using real-time quantitative PCR and the 2-(Delta Delta C(T)) method. Methods 25: 402-408, 2001.

13. Berman DM, Karhadkar SS, Hallahan AR, Pritchard JI, Eberhart CG, Watkins DN, Chen JK, Cooper MK, Taipale J, Olson JM and Beachy PA: Medulloblastoma growth inhibition by hedgehog pathway blockade. Science 297: 1559-1561, 2002.

14. Berman DM, Karhadkar SS, Maitra A, Montes De Oca R, Gerstenblith MR, Briggs K, Parker AR, Shimada Y, Eshleman JR, Watkins DN and Beachy PA: Widespread requirement for Hedgehog ligand stimulation in growth of digestive tract tumours. Nature 425: 846-851, 2003 .

15. Karhadkar SS, Bova GS, Abdallah N, Dhara S, Gardner D, Maitra A, Isaacs JT, Berman DM and Beachy PA: Hedgehog signalling in prostate regeneration, neoplasia and metastasis. Nature 431: 707-712, 2004.

16. Thayer SP, di Magliano MP, Heiser PW, Nielsen CM, Roberts DJ, Lauwers GY, Qi YP, Gysin S, Fernández-del Castillo C, Yajnik V, et al: Hedgehog is an early and late mediator of pancreatic cancer tumorigenesis. Nature 425: 851-856, 2003.

17. Watkins DN, Berman DM, Burkholder SG, Wang B, Beachy PA and Baylin SB: Hedgehog signalling within airway epithelial progenitors and in small-cell lung cancer. Nature 422: 313-317, 2003.

18. Yoo YA, Kang MH, Lee HJ, Kim BH, Park JK, Kim HK, Kim JS and Oh SC: Sonic hedgehog pathway promotes metastasis and lymphangiogenesis via activation of Akt, EMT, and MMP-9 pathway in gastric cancer. Cancer Res 71: 7061-7070, 2011.

19. Mill P, Mo R, Hu MC, Dagnino L, Rosenblum ND and Hui CC: Shh controls epithelial proliferation via independent pathways that converge on N-Myc. Dev Cell 9: 293-303, 2005.

20. Eilers M and Eisenman RN: Myc's broad reach. Genes Dev 22: 2755-2766, 2008.

21. Javelaud D, Pierrat MJ and Mauviel A: Crosstalk between TGF- $\beta$ and hedgehog signaling in cancer. FEBS Lett 586: 2016-2025, 2012.

22. Yanai K, Nakamura M, Akiyoshi T, Nagai S, Wada J, Koga K, Noshiro H, Nagai E, Tsuneyoshi M, Tanaka M and Katano M: Crosstalk of hedgehog and Wnt pathways in gastric cancer. Cancer Lett 263: 145-156, 2008.

23. Riobo K, Lu X, Ai GM, Haines CP and Emerson CP Jr: Phosphoinositide 3-kinase, Akt are essential for Sonic Hedgehog signaling. Proc Natl Acad Sci USA 103: 4505-4510, 2006. 